\title{
DATOS PALINOLOGICOS DEL BAYOCIANO (FORMACIÓN CURA NIYEU) DE LA SIERRA DE CHACAI CO, CUENCA NEUQUINA, ARGENTINA
}

\author{
OSCAR GONZALEZ-AMICON \\ Museo Argentino de Ciencias Naturales "Bernardino Rivadavia", \\ Buenos Aires \\ WOLFGANG VOLKHEIMER \\ Museo Argentino de Ciencias Naturales "Bernardino Rivadavia", \\ Buenos Aires, y CONICET
}

\begin{abstract}
"Palynological data from the Bayocian Cura Niyeu Fm. of Sierra de Chacai Có, Neuquén Basin, Argentina". - In marine strata with Sonninia ssp. and Otoites spp., 5 microfloristic assemblages have been identified. More than 60 terrestrial and 8 marine species of palynomorphs belong to the following genera: Alisporites, Anapiculatisporites, Antulsporites, Araucariacites, Baculatisporites, Biretisporites, Callialasporites, Cerebropollenites, Classopollis, Concavisporites, Converrucosisporites, Cycadopites, Deltoidospora, Dictyophyllidites, Duplexisporites, Equisetosporites, Gleicheniidites, Inapertisporites, Interulobites, Ischyosporites, Leptolepidites, Lycopodiumsporites, Marattisporites, Microcachryidites, Monosulcites, Nevesisporites, Perinopollenites, Peromonolites, Phrixipollenites, Podocarpidites, Staplinisporites, Stereisporites, Taurocusporites, Todisporites, Trisaccites, Uvaesporites, Verrucosisporites, Vitreisporites, Baltisphaeridium, Campenia, Cymatiosphaera, Leiosphaeridia, Lancettopsis, Micrhystridium, Pleurozonaria, Veryhachium. The microflora is compared with other palynomorph assemblages from the Middle and Upper Jurassic of the Neuquén Basin and other regions.
\end{abstract}

\section{INTRODUCCION}

Los estudios palinológicos en el Jurásico de la Sierra de Chacai Có comenzaron con el análisis sistemático de microfloras de las formaciones Lajas (VOLKHEIMER, 1972), Sierra Chacai Có y Los Molles (VOLKHEIMER, 1974). Un perfil estratigráfico detallado de la Fm. Cura Niyeu (Bayociano), objeto del presente trabajo, con la ubicación de los fósiles guía de la macrofauna y asimismo la ubicación de las microfloras, se halla en VOLKHEIMER (1973). Dado el tratamiento relativamente completo en la publicación citada, se prescinde aquí de una parte estratigráfica, dejando aclarado que en su localidad tipo, la Fm. Cura Niyeu se apoya sobre las lutitas da la Fm. Los Molles (Toarciano-Aaleniano) y transita hacia arriba a la facies deltaica de la Fm. Lajas (Bayociano superior, Batoniano y Caloviano inferior).

\section{METODOS, TECNICAS Y MATERIALES}

El trabajo de campo fue realizado por uno de nosotros (W.V.) en la localidad tipo de la Fm. Cura Niyeu (Cañadón de Charahuilla). 


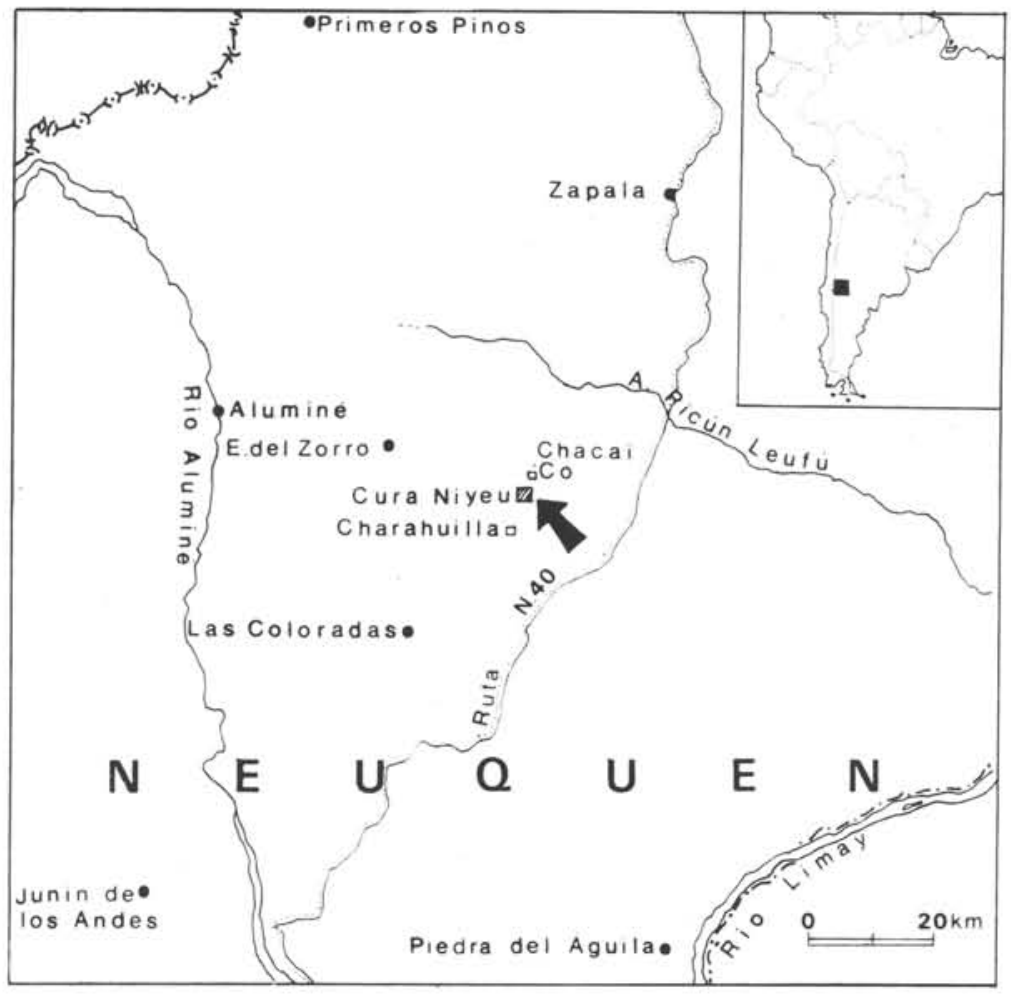

Fig. 1 - Mạ́a de ubicación.

Las muestras palinológicas estudiadas están archivadas en la palinoteca de la División Paleobotánica del Museo Argentino de Ciencias Naturales "Bernardino Rivadavia" bajo los números $814,838,839,857$ y 860 BAPB. La extracción fisicoquímica de las muestras se realizó según las normas expuestras en VOLKHEIMER \& MELENDI (1976). El estudio se efectuó con el microscopio Leitz Ortholux No 762978 de la mencionada División.

Cuadro 1: Posición estratigráfica de las muestras palinológicas

\begin{tabular}{|c|c|c|c|c|}
\hline $\begin{array}{l}\text { Asociación } \\
\text { microflorística }\end{array}$ & $\begin{array}{l}\text { Metros sobre base } \\
\text { Fm. Cura Niyeu }\end{array}$ & Litología & $\begin{array}{l}\text { No lab. } \\
\text { palin. }\end{array}$ & $\begin{array}{l}\text { NO de } \\
\text { campo }\end{array}$ \\
\hline$E$ & 80 & $\begin{array}{l}\text { Calcarenita } \\
\text { limolítica }\end{array}$ & 860 & CC16 \\
\hline D & 74 & $\begin{array}{l}\text { Limolita } \\
\text { calcarea- } \\
\text { areniscosa }\end{array}$ & 839 & $\mathrm{CC} 15$ \\
\hline C & 58 & $\begin{array}{l}\text { Calcarenita } \\
\text { gris }\end{array}$ & 838 & $\mathrm{CC} 12$ \\
\hline B & 48 & $\begin{array}{l}\text { Marga } \\
\text { gris }\end{array}$ & 857 & CC 9 \\
\hline A & 5 & $\begin{array}{l}\text { Calcarenita } \\
\text { gris }\end{array}$ & 814 & CC 2 \\
\hline
\end{tabular}


GONZALES-AMICON, O. \& VOLKHEIMER, W.

\section{LISTA TAXONOMICA DE ESPECIES IDENTIFICADAS}

\section{MIOSPORAS AZONOTRILETES LISAS (Infraturma LAEVIGATI) \\ Deltoidospora australis (Couper) Pocock 1970}

Deltoidospora minor (Couper) Pocock 1970

Deltoidospora neddeni Pflug 1953

Deltoidospora sp.

Todisporites major Couper 1958

Dictyophyllidites mortoni (de Jersey) Play. ford \& Dettman 1965

Dictyophyllidites sp.

Biretisporites sp.

Concavisporites semiangulatus Menéndez 1968

Concavisporites sp.

Stereisporites sp.

\section{MIOSPORAS AZONOTRILETES \\ CON ESCULTURA UNITARIA \\ (Infraturma APICULATI)}

Verrucosisporites cf. opimus Manum 1962

Verrucosisporites varians Volkheimer 1972

Verrucosisporites sp.

Baculatisporites sp.

Uvaesporites minimus Volkheimer 1968

Uvaesporites $\mathrm{sp}$.

Leptolepidites macroverrucosus Schulz 1967

Leptolepidites major Couper 1958

Anapiculatisporites dawsonensis Reiser \& Williams 1969

Convernucosisporites sp.

\section{MIOSPORAS ZONOTRILETES CON ENGROSAMIENTOS INTERRADIALES \\ UNICAMENTE (Infraturma TRICRASSATI)}

Gleicheniidites argentinus Volkheimer 1972

$$
\begin{aligned}
& \text { MIOSPORAS AZONOTRILETES } \\
& \text { CON ESCULTURA CONTINUADA } \\
& \text { (Infraturma MURORNATI) }
\end{aligned}
$$

\section{Lycopodiumsporites austroclavatidites}

(Cookson) Potonié 1956

Lycopodiumsporites semimuris (Danzé-Corsin \& Laveine) Reiser \& Williams 1969

sin \& Laveine) Reiser \& Williams 1969

Staplinisporites caminus (Balme) Pocock 1962

Ischyosporites marburgensis de Jersey 1963
Palinologia de la Formación Cura Niyeu. .. p. 108-115

\section{MIOSPORAS ZONOTRILETES \\ CON ENGROSAMIENTO ECUATORIAL \\ (Infraturma CINGULATI)}

Antulsporites saevus (Balme) Archangelsky \& Gamerro 1966

Interulobites sp.

Duplexisporites sp.

Nevesisporites vallatus? de Jersey \& Paten 1964

Nevesisporites sp.

Taurocusporites sp.

\section{MIOSPORAS MONOLETES \\ (Turma MONOLETES)}

Peromonolites sp.

Marattisporites cf. scabratus Couper 1958

\section{GRANOS DE POLEN SACADOS (Turma SACCITES)}

Callialasporites dampieri (Balme) Dev 1961 Callialasporites microvelatus Schulz 1966

Callialasporites segmentatus (Balme) Srivastava 1963

Callialasporites turbatus (Balme) Schulz 1967

Callialasporites $\mathrm{sp}$.

Vitreisporites pallidus (Reissinger) Nilsson 1958

Vitreisporites sp.

Alisporites sp.

Phrixipollenites cf. otagoensis (Couper) Haskell 1968

Phrixipollenites sp.

Podocarpidites verrucosus Volkheimer 1972

Podocarpidites sp.

Microcachryidites antarcticus Cookson 1947

Microcachryidites castellanosii Menéndez 1968

Microcachryidites sp.

Trisaccites microsaccatus (Couper) Couper 1960

\section{GRANOS DE POLEN INAPERTURADOS \\ (Turma ALETES)}

Inaperturopollenites indicus Srivastava 1966 Inaperturopollenites $\mathrm{sp}$.

Araucariacites australis Cookson 1947

Araucariacites fissus Reiser \& Williams 1969 Araucariacites pergranulatus Volkheimer 1968

Araucariacites sp.

Inapertisporites sp. 
GRANOS DE POLEN PORADOS (Turma POROSES)

Perinopollenites elatoides Couper 1958 Classopollis intrareticulatus Volkheimer 1972 Classopollis simplex (Danzé-Corsin \& Laveine) Reiser \& Williams 1969

Classopollis torosus (Reissinger 1950, Couper 1958) emend. Burger 1965

Classopollis sp.

\section{GRANOS DE POLEN COLPADOS (Turma PLICATES)}

Cycadopites granulatus (de Jersey) de Jersey 1964

Cycadopites nitidus (Balme) de Jersey 1964

Cycadopites sp.

Monosulcites sp.

Cerebropollenites cf. macroverrucosus (Thiergart) Schulz 1967

Equisetosporites caichigüensis Volkheimer \& Quattrocchio 1975

\section{GRUPO ACRITARCHA}

SUBGRUPO ACANTHOMORPHITAE

Downie, Evitt \& Sarjeant 1963

Baltisphaeridium cf. debilispinum Wall \& Downie 1963

Micrhystridium sp.

\section{SUBGRUPO HERKOMORPHITAE}

Downie, Evitt \& Sarjeant 1963

Cymatiosphaera sp.

\section{SUBGRUPO POLYGONOMORPHITAE \\ Downie, Evitt \& Sarjeant 1963}

Veryhachium sp.

\section{SUBGRUPO SPHAEROMORPHITAE \\ Downie, Evitt \& Sarjeant 1963}

\section{Campenia sp.}

Lancettopsis sp.

Leiosphaeridia hyalina (Deflandre) Downie 1957

Leiosphaeridia sp.

\section{MISCELANEA}

Pleurozonaria sp.

\section{ASPECTOS ESTADISTICOS}

A nivel supragenérico, se presentan en las cinco asociaciones microflorísticas estudiadas, los grupos de palinomorfos representados en el Cuadro 2.

Cuadro 2: Frecuencia de grupos supragenéricos en recuentos de 200 ejemplares de cinco asociaciones microflorísticas de la Fm. Cura Niyeu, Bayociano, en su localidad tipo.

\begin{tabular}{|l|c|c|c|c|c|}
\hline \multirow{2}{*}{\begin{tabular}{|c|} 
G RUPOS \\
SUPRAGENÉRICOS
\end{tabular}} & A & B & C & D & E \\
\cline { 2 - 6 } & 1,5 & 1,0 & 3,0 & 2,5 & 2,0 \\
\hline Esporas triletes y monoletes & 11,5 & 16,0 & 24,5 & 13,5 & 14,5 \\
Granos de polen sacados & 16,0 & 8,5 & 14,0 & 7,0 & 9,5 \\
Granos de polen inaperturados & 0,5 & 1,0 & + & 2,5 & 2,0 \\
Granos monosulcados & - & - & + & - & - \\
Granos de polen poliplicados & 69,5 & 54,5 & 55,0 & 66,0 & 61,0 \\
Granos de polen monoporados & 1,0 & 19,0 & 3,5 & 8,5 & 11,0 \\
Acritarcos & (+: presente solamente, fuera del recuento.) \\
\hline \multicolumn{7}{|l|}{} \\
\hline
\end{tabular}




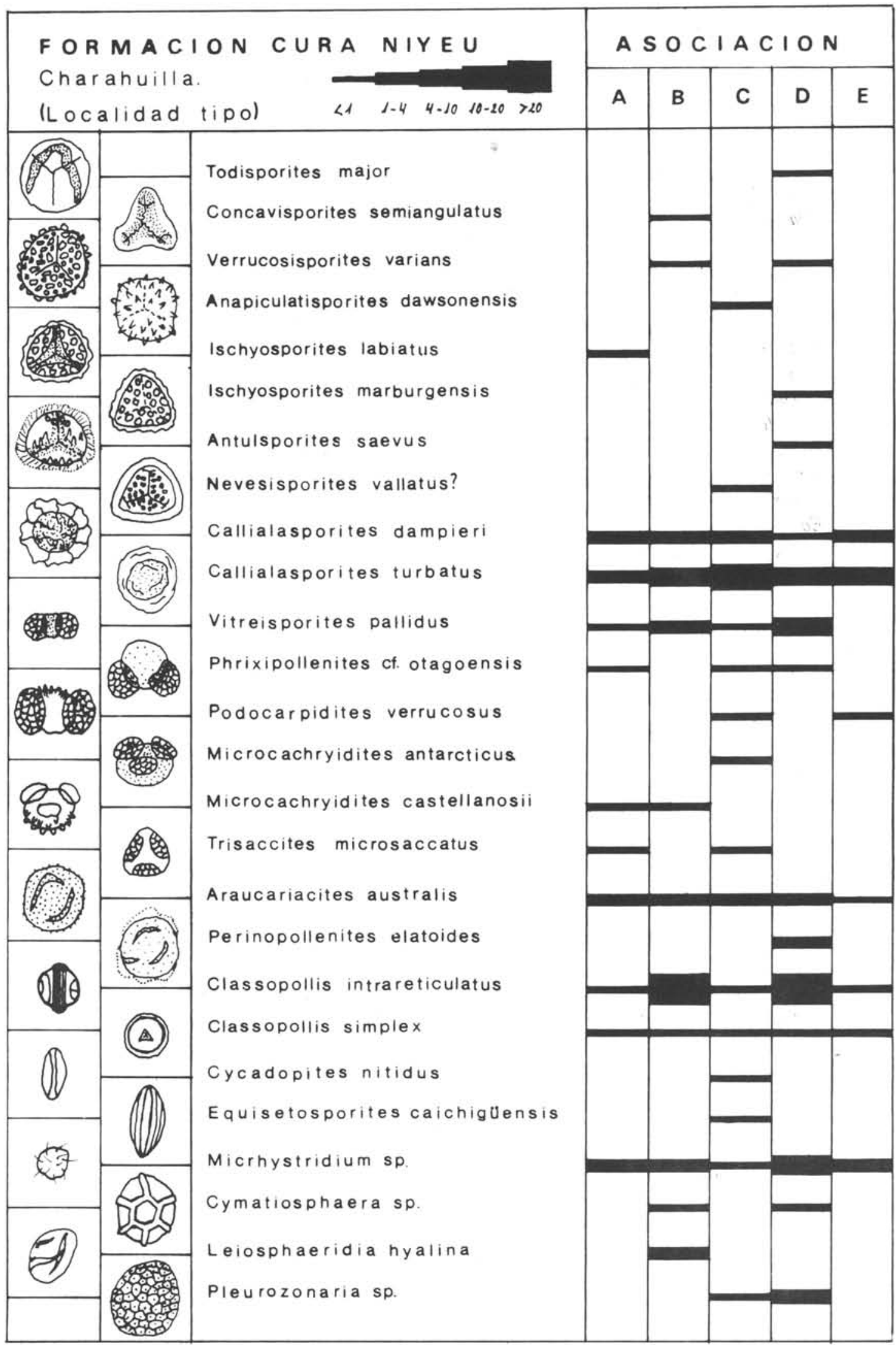


En todas las asociaciones predominan los granos monoporados, (Classopollis spp.) con $54,5-69,5 \%$ del espectro. Otro grupo supragenérico numéricamente importante es el de los granos sacados $(11,5-24,5 \%)$, representado sobre todo, por cuatro especies de Callialasporites y por Vitreisporites. Entre los granos inaperturados $(7,0-16,0 \%)$, se destacan Inaperturapollenites sp. y Araucariacites australis. Las formas agrupadas en los recuentos en el género Inaperturopollenites incluyen, posiblemente, acritarcos esféricos más o menos levigados que carecen, a nivel óptico, de rasgos que los distingan de ese género. Los granos monosulcados (hasta 2,5\%), son poco frecuentes; los poliplicados aparecen ocasionalmente, en una sola asociación (C), y las especies marinas, presentes en todas las asociaciones (principalmente acritarcos), abarcan entre 1 y $19 \%$ del espectro.

En el Cuadro 3 se presenta la distribución de los taxones de palinomorfos dispersos más característicos en las asociaciones microflorísticas de la Formación Cura Niyeu en su localidad tipo.

\section{EDAD DE LA MICROFLORA}

Gran parte de los palinomorfos presentes en la microflora de la Fm. Cura Niyeu tiene una amplia distribución estratigráfica en formaciones jurásicas y cretácicas de la Cuenca Neuquina y otras regiones del mundo. No consideraremos tales taxones para fines de datación; la evaluación estadística de ellos proporciona, en cambio, valiosos datos paleoecológicos (VOLKHEIMER, 1978). Entre las formas de biocrón restringido se halla Ischyosporites marburgensis de Jersey, que se distribuye en el Jurásico inferior de Australia (DE JERSEY, 1963) y nunca se ha observado en estratos más recientes que el Bayociano.

La primera aparición del género Callialasporites, que se presenta en nuestro material con las especies C. dampieri, C. microvelatus, $C$. segmentatus y $C$. turbatus, se observa en va-

Cuadro 3: Distribución de taxones elegidos de palinomorfos dispersos en las asociaciones microflorísticas de la Formación Cura Niyeu (Bayociano) en su localidad tipo. rias cuencas sedimentarias del mundo a partir del Toarciano superior basal (estratos con Phymatoceras del grupo copiapense en el caso de la Cuenca Neuquina), distribuyéndose a través de todo el Jurásico medio y superior y el Cretácico inferior. De la presencia de las ešpecies mencionadas resulta, por las razones expuestas, que la edad máxima indicada por la microflora es el Toarciano superior, y la edad mínima el Bayociano.

Otra de las especies presentes Microcachryidites castellanosii, observada en un principio solo en estratos del Caloviano inferior, tiene según estudios recientes, un biocrón más amplio, llegando al Oxfordiano (VOKHEIMER \& MORONI, 1981) por un lado, y al Lías (ARGUIJO et al., en prensa, este tomo) por el otro. Tal amplia distribución jurásica está de acuerdo con lo observado en Australia occidental (FILATOFF, 1975).

En el Cuadro 4 se puede observar cuales especies de la Fm. Cura Niyeu se presentan en otras microfloras jurásicas de la Cuenca Neuquina.

\section{CONCLUSIONES}

1. En la localidad tipo de la Formación Cura Niyeu (Cañadón de Charahuilla) se hallaron cinco asociaciones microflorísticas con un total de 69 especies terrestres y 9 especies marinas.

2. El ambiente de depositación es nerítico.

3. El estudio estadístico de las asociaciones de palinomorfos demuestra el predominio neto del género Classopollis en todas ellas, reflejo de una abundante vegetación costera de Cheirolepidiaceae.

4. La composición de la microflora indica como edad máxima el Toarciano superior, y como edad mínima el Bayociano. Esta última edad es la indicada por los amonites asociados a la microflora (Sonninia spp., Otoites spp.).

\section{AGRADECIMIENTOS}

Se agradece sinceramente al Museo Argentino de Ciencias Naturales "Bernardino Rivadavia" por haber proporcionado un vehículo apto para los trabajos en campaña y por las facilidades de laboratorio; al CONICET y a la SECYT por el apoyo económico brindado. 
Cuadro 4: Presencia de especies de la microflora bayociana de la Formación Cura Niyeu en otras microfloras del Jurásico medio y superior de la Cuenca Neuquina.

AN: Fm. Arcillas Negras, Bayociano (Volkheimer 1968); LA: Fm. Lajas, Caloviano (Volkheimer 1972); AU: Fm. Auquinco, Oxfordiano (Volkheimer \& Moroni 1981); VM: Fm. Vaca Muerta, Titoniano inferior (Vokheimer \& Quattrocchio 1975).

\begin{tabular}{|c|c|c|c|c|}
\hline ESPECIES & AN & LA & $\mathrm{AU}$ & VM \\
\hline Anapiculatisporites dawsonensis & & & cf. & cf. \\
\hline Antulsporites saevus & & & + & \\
\hline Araucariacites australis & + & + & & + \\
\hline Araucariacites pergranulatus & + & + & + & + \\
\hline Callialasporites dampieri & + & + & + & + \\
\hline Callialasporites microvelatus & & & + & + \\
\hline Callialasporites segmentatus & + & & + & \\
\hline Classopollis intrareticulatus & & + & + & + \\
\hline Classopollis simplex & + & & + & + \\
\hline Classopollis torosus & cf. & & + & \\
\hline Concavisporites semiangulatus & & + & & \\
\hline Cycadopites granulatus & + & + & + & + \\
\hline Cycadopites nitidus & + & + & + & \\
\hline Deltoidospora australis & + & & + & \\
\hline Deltoidospora minor & + & & & \\
\hline Deltoidospora neddeni & + & + & & \\
\hline Dictyophyllidites mortoni & + & & & cf. \\
\hline Equisetosporites caichigüensis & & & & + \\
\hline Gleicheniidites argentinus & & + & & \\
\hline Inaperturopollenites indicus & + & $c f$. & & \\
\hline Ischyosporites labiatus & + & & & \\
\hline Ischyosporites marburgensis & + & & & \\
\hline Leisosphaeridia hyalina & & & cf. & \\
\hline Leptolepidites macroverrucosus & & & cf. & cf. \\
\hline Leptolepidites major & & & cf. & \\
\hline Lycopodiumsporites austroclavatidites & + & + & & + \\
\hline Marattisporites cf. scabratus & & + & & + \\
\hline Microcachryidites antarcticus & + & & & + \\
\hline Microcachryidites castellanosii & & + & + & \\
\hline Perinopollenites elatoides & & + & & \\
\hline Phrixipollenites cf. otagoensis & + & & + & \\
\hline Podocarpidites verrucosus & & + & cf. & \\
\hline Todisporites major & + & + & & \\
\hline Trisaccites microsaccatus & & & & + \\
\hline Uvaesporites minimus & + & & + & cf. \\
\hline Verrucosisporites cf. opimus & + & & & \\
\hline Verrucosisporites varians & & + & & \\
\hline Vitreisporites pallidus & + & + & + & \\
\hline
\end{tabular}




\section{BIBLIOGRAFIA}

ARGUiJO, M.H., VOLKHEIMER, W. \& ROSENFELD, U. - 1981 - Estudio palinológico de la Formación Piedra Pintada, Jurásico inferior de la Cuenca Neuquina (Argentina). Actas III Reunião de Paleobotánicos e Palinólogos. São Paulo. En prensa, este tomo.

DE JERSEY, N.J. - 1963 - Jurassic spores and pollen grains from the Marburg Sandstone. Geol. Surv. Queensland Pub. 313:1-15.

FILATOFF, J. - 1975 - Jurassic Palynology of the Perth Basin, Western Australia. Paleontographica Abt. B-154: $1-113$, Stuttgart.

VOLKHEIMER, W. - 1968 - Esporas y granos de polen del Jurásico de Neuquén (República Argentina). I. Descripciones sistemáticas. Ameghiniana 5(9): 330-370.

VOLKHEIMER, W. - 1972 - Estudio palinológico de un carbón caloviano de Neuquén y consideraciones sobre los paleoclimas jurásicos de la Argetina. Rev. Museo de La Plata 6 (n. s.), Paleontología 40: 101-157.

VOLKHEIMER, W. - 1973 - Palinología Estratigráfica del Jurásico de la Sierra de Chacai Có y adyacencias (Cuenca Neuquina, República Argentina) I. Estratigrafía. Ameghiniana 10(2): 105-131.

VOLKHEIMER, W. - 1974 - Palinología Estratigrafica del Jurásico de la Sierra de Chacai Có y adyacencias (Cuenca Neuquina, República Argentina). II Descripción de los palinomorfos de las formaciones Sierra Chacai Có (Pliensbachiano) y Los Molles (Toarciano-Aaleniano). Ameghiniana 11(2): 135-172.

VOLKHEIMER, W. - 1978 - Microfloras fósiles. Relatorio Geología y Recursos Naturales del Neuquén. VII Congreso Geológico Argentino: 193-207.

VOLKHEIMER, W. \& MELENDI, D.L. - 1976 - Palinomorfos como fósiles guía. Tercera parte: Técnicas de laboratorio palinológico. Revista Minera, Geol. y Mineral., Soc. Argent. Min. y Geol. Tomo 34(1/2): 19-30.

VOLKHEIMER, W. \& MORONI, A.M. - 1981 - Datos Palinológicos de la Formación Auquinco. Jurásico superior de la Cuenca Neuquina, Argentina. Actas VIII Congreso Geológico Argentino; IV: 795-812.

VOLKHEIMER, W. \& QUATTROCCHIO, M. - 1975 - Palinología estratigrdfica del Titoniano (Formación Vaca Muerta) en el área de Caichigüe (Cuenca Nequina). Parte A: especies terrestres. Ameghiniana 12(3): 193-241. 